\title{
Mid-infrared Raman Soliton Generation in Chalcogenide Microwire
}

\author{
Imtiaz Alamgir ${ }^{1 *}$, Md Hosne Mobarok Shamim ${ }^{1}$, WAgner CORRer $^{2}$, Younès \\ MESSADdEQ ${ }^{2}$ AND MARTIN ROCHETTE ${ }^{1}$
}

\author{
${ }^{1}$ Department of Electrical and Computer Engineering, McGill University, Montreal (QC), Canada \\ ${ }^{2}$ Centre d'Optique, Photonique et Laser, Université Laval, Québec (QC), Canada \\ *Corresponding author: md.alamgir@mail.mcgill.ca
}

\begin{abstract}
Emerging applications in the mid-infrared (MIR) stimulate the growth and development of novel optical light sources. Soliton self-frequency shift (SSFS) in soft glass fiber currently show great potential as an efficient approach towards the generation of broadly tunable femtosecond pulses in the MIR. In this work, we demonstrate highly efficient tunable soliton source based on SSFS in chalcogenide glass. We show a simple and fully fiberized system to generate these continuously tunable Raman solitons over a broad spectral range of 2.047-2.667 $\mu \mathrm{m}$, which consumes no more than $87 \mathrm{pJ}$ per pulse. The spectral measurements suggest that the generated pulses are as short as $62 \mathrm{fs}$ with a maximum power conversion efficiency of $43 \%$. This result is realized thanks to an $8 \mathrm{~cm}$ long $\mathrm{As}_{2} \mathrm{~S}_{3}$ microstructure optical fiber tapered into a microwire. Thanks to their broad transparency, their high nonlinearity and their adjustable chromatic dispersion, chalcogenide microwires are promising components for the development of compact, low power consumption and highly efficient MIR optical sources.
\end{abstract}

The growing interest for the use of mid-infrared (MIR) in engineering and fundamental sciences have intensified the development of novel light sources. In addition to conventional gain media with electronic transitions such as rare-earth doped glasses and crystals, nonlinear wavelength converters such as optical parametric oscillators and difference frequency generators are promising means to reach the MIR [1]. Solid-state optical systems have the capacity to generate short and high energy pulses for technologies such as medical surgery, material processing, strongfield physics and attosecond science [2-4]. On the other hand, compact and low power consumption sources capable to generate femtosecond pulses are desirable for spectroscopic and sensing applications such as breath analysis, environmental monitoring, food quality control and trace gas detection [5-8].

Compactness, robustness, and low power consumption are a common quality of many fiber-based light sources. With the use of optical fibers, nonlinear wavelength conversion techniques include supercontinuum generation [9-11], parametric sideband conversion [12-14] and soliton self-frequency shift (SSFS) [15]. Among these methods, SSFS is of particular interest since it can generate tunable and ultrashort pulses from a pulsed light source paired with a suitable nonlinear fiber. In contrast with other wavelength conversion techniques, it requires no additional probe laser or temporal synchronization, and the power spectral density of the SSFS pulse is concentrated into a narrow, targeted set of wavelengths.

SSFS has been investigated in standard silica fibers, where the maximum wavelength shift of solitons is limited to $\sim 2 \mu \mathrm{m}$ by the transmission loss of silica [16-18]. For SSFS applications at wavelengths longer than $2 \mu \mathrm{m}$, soft glass fibers such as chalcogenide, fluoride, and tellurite maintain low transmission losses thanks to their low phonon energies. For example, Tang et al. demonstrated solitons that are tunable over the spectral range of 2$4.3 \mu \mathrm{m}$ in a $2 \mathrm{~m}$ long $\mathrm{InF}_{3}$ fiber from an input pump energy of $120 \mathrm{~nJ}$ [19]. Duval et al. generated solitons tunable from a wavelength of $2.8 \mu \mathrm{m}$ to $3.6 \mu \mathrm{m}$ from a maximum energy of $37 \mathrm{~nJ}$ injected into a $22 \mathrm{~m}$ long $\mathrm{ZrF}_{4}$ fiber [20]. These hybrid fiber/free-space systems generate soliton of relatively large energy and find use in high power applications. For low power consumption wavelength converters that operate from low energy soliton, highly nonlinear medium is desirable [21]. Koptev et al. have demonstrated SSFS in $0.5 \mathrm{~m}$ long microstructured tellurite fiber tunable within a wavelength range of 2.25-2.65 $\mu \mathrm{m}$ from an input energy of $3 \mathrm{~nJ}$ [22]. However, the development of more compact and portable systems requires a fully fiberized architecture capable to operate from pump energies at the scale of picojoules.

Chalcogenide (ChG) glasses (e.g., $\mathrm{As}_{2} \mathrm{Se}_{3}, \mathrm{As}_{2} \mathrm{~S}_{3}$ ) are excellent candidates for the fabrication of SSFS-based wavelength converters that are compact and consume low power. ChG glasses have an exceptionally high nonlinear refractive index, nearly three orders of magnitude beyond that of silica glass and their MIR optical transmission spectrum extends to wavelengths up to $12 \mu \mathrm{m}$ [23, 24]. Cheng et al. demonstrated SSFS in an $\mathrm{AsSe}_{2}-\mathrm{As}_{2} \mathrm{~S}_{5}$ fiber from a solid-state pump source centered at a wavelength of $2.8 \mu \mathrm{m}$ [25]. A frequency detuning of $620 \mathrm{~nm}(19.43 \mathrm{THz})$ was obtained for a pump power of $144 \mathrm{~mW}$, or pulse energy of $1.8 \mathrm{~nJ}$. Since this SSFS was observed in the context of supercontinuum generation, the generated fundamental soliton was not clearly distinguishable and the power conversion efficiency (PCE) to this specific soliton remained negligible. To improve the PCE and minimize the energy required to trigger SSFS, the enhanced nonlinear optical effect of tapered ChG fibers is of great use [26, 27]. ChG tapers allows for engineerable chromatic dispersion and thereby enable an easy control of the soliton center wavelength [21, 28]. Al-Kadry et al. 
performed theoretical investigation of SSFS in $\mathrm{As}_{2} \mathrm{Se}_{3}$ microwires where they predicted SSFS tunable from $2.10-2.75 \mu \mathrm{m}$ with maximum PCE $=95 \%$ at a pump energy of $2.37 \mathrm{pJ}$ [29]. However, to this date, there has been no experimental demonstration of a tunable soliton source realized via SSFS in ChG microwire.
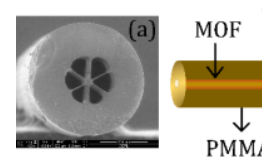

Transition section

(b)

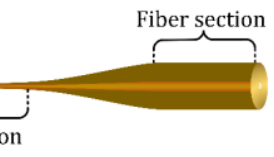

Fig. 1. (a) Scanning electron microscope image of the $\mathrm{As}_{2} \mathrm{~S}_{3}$ suspended core microstructured fiber (fiber diameter: $213 \mu \mathrm{m}$ ). (b) Schematic of the tapered fiber.

In this work, we demonstrate the first experimental realization of high efficiency SSFS in ChG microwires. The wavelength converter is widely tunable within the spectral range of $2.047-2.667 \mu \mathrm{m}$, providing an SSFS detuning of up to $42.3 \mathrm{THz}(729 \mathrm{~nm})$ from a pump wavelength of $1.938 \mu \mathrm{m}$ and an energy of $<87 \mathrm{pJ}$ per pulse. The core of the wavelength converter is an $8 \mathrm{~cm}$ long nonlinearity and dispersion engineered $\mathrm{As}_{2} \mathrm{~S}_{3}$ microwire, tapered from a suspended core microstructured fiber (MOF). The PCE in this experiment is exceptional, the largest ever demonstrated in ChG fiber, with a maximum of $43 \%$ and a value maintained above $20 \%$ over the whole tuning range. The proposed wavelength converter demonstrates the feasibility to develop light sources that are compact, with low power consumption, and broadly tunable in the MIR.

The nonlinear medium used in this experiment is an $\mathrm{As}_{2} \mathrm{~S}_{3} \mathrm{MOF}$ with a diameter of $213 \mu \mathrm{m}$. Fig. 1 (a) shows the scanning electron microscope image of the MOF's cross-section. The suspended core has a diameter of $13.5 \mu \mathrm{m}$ and is surrounded by six air holes. The fiber is multimode with a V-number of 47.7. Prior to fabrication, the MOF is coated with poly-methyl methacrylate (PMMA) as an extra layer that brings robustness to the microwire while preserving the optical mode in the $\mathrm{As}_{2} \mathrm{~S}_{3}$-air material. Fig .1 (b) depicts a schematic of the taper including the microwire section where the nonlinear process occurs, two transition sections, and two fiber sections for efficient coupling from an input fiber or to an output fiber. The group velocity dispersion (GVD) parameter $\left(\beta_{2}\right)$ of the microwire can be engineered into zero, anomalous, or normal dispersion at the pump wavelength by choosing an appropriate microwire section diameter. During fabrication, the central portion of the MOF is flame-brush tapered into a microwire section while simultaneously monitoring the chromatic dispersion up until a desired level of $\beta_{2}=-1 \mathrm{ps}^{2} / \mathrm{m}$ [30]. After tapering, the microwire section reaches a length of $8 \mathrm{~cm}$ while the fiber section and the transition sections reach a length of $1 \mathrm{~cm}$ and $2 \mathrm{~cm}$, respectively.

Fig. 2 (a) and (b) show the calculated GVD and waveguide nonlinearity $(\gamma)$ of the microwire, respectively. COMSOL Multiphysics was used to calculate the GVD by performing a full vectorial finite-element method analysis of the fiber geometry. For the designed GVD at the pump wavelength of $1.938 \mu \mathrm{m}$, the core diameter of the MOF reduces to $1.34 \mu \mathrm{m}$. With this core diameter, strong modal confinement due to the large refractive index contrast between $\mathrm{As}_{2} \mathrm{~S}_{3}\left(\mathrm{n}_{2}=2.7 \times 10^{-18} \mathrm{~m}^{2} / \mathrm{W}\right)$ and air leads to a waveguide nonlinearity of $\gamma=9.14 \mathrm{~W}^{-1} \mathrm{~m}^{-1}$. The fiber section and the transition section have a negligible effect on the pump pulse properties.
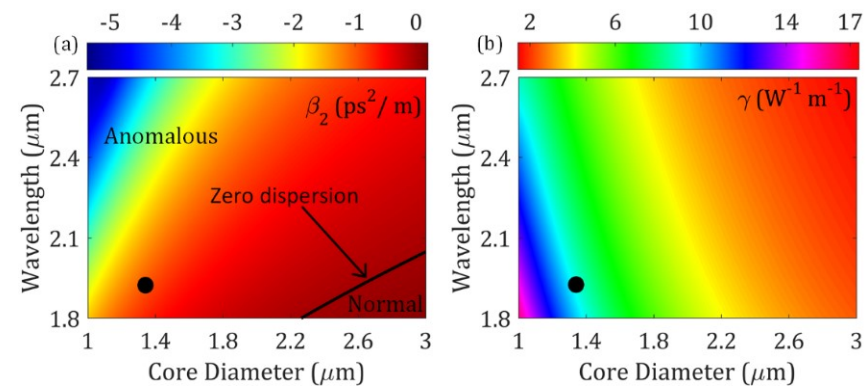

Fig. 2. (a) GVD of the microwire as a function of wavelength and core diameter. The zero-dispersion line separates the anomalous and normal dispersion regions. (b) Waveguide nonlinearity of the microwire. The black dot in both figures indicate the designed microwire.

The input facet of the taper is cleaved and aligned to a single mode fiber (SMF-28). The output side of the taper is aligned to one end of a $20 \mathrm{~cm}$-long single mode step index $\mathrm{As}_{2} \mathrm{Se}_{3}$ fiber (Coractive IRT-SE-6/170) with core/clad diameters of $6 \mu \mathrm{m} / 170 \mu \mathrm{m}$ and $\mathrm{NA}=0.16$. Both ends of the taper are then mechanically spliced using UV-cured epoxy. The total insertion loss of the SMF-taper-ChG fiber assembly at a wavelength of $1.938 \mu \mathrm{m}$ is $3.4 \mathrm{~dB}$. From this loss, $0.3 \mathrm{~dB}$ and $0.02 \mathrm{~dB}$ are due to Fresnel reflection at the $\mathrm{As}_{2} \mathrm{~S}_{3}$ - silica and $\mathrm{As}_{2} \mathrm{~S}_{3}-\mathrm{As}_{2} \mathrm{Se}_{3}$ interfaces, respectively, $0.5 \mathrm{~dB}$ is due to propagation loss in the microtaper, $1 \mathrm{~dB}$ is due to mode-mismatch and the balance is attributed to fiber misalignment. For spectral analysis, the other end of the $\mathrm{As}_{2} \mathrm{Se}_{3}$ fiber is connected to a Yokogawa AQ6376 optical spectrum analyzer (OSA). The short length of the $\mathrm{As}_{2} \mathrm{Se}_{3}$ fiber ensures that the low level of added chromatic dispersion is negligible for the sampled soliton.
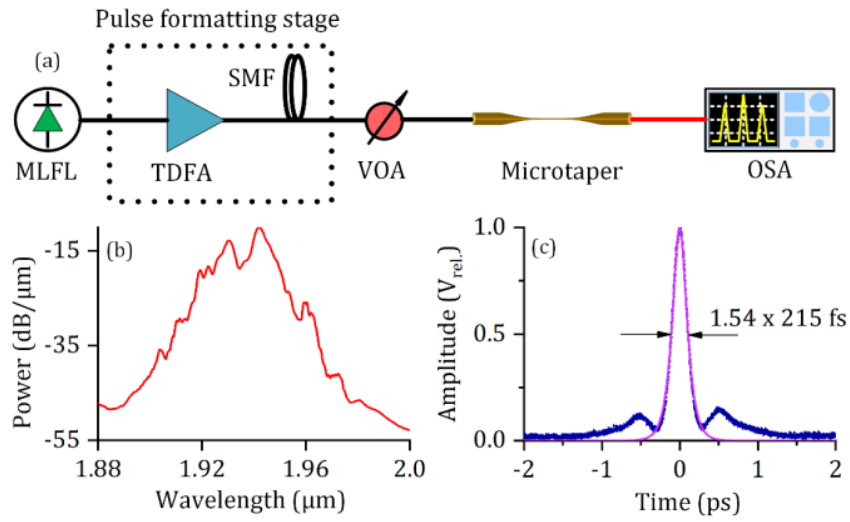

Fig. 3. (a) Schematic of the experimental setup. MLFL, mode- locked fiber laser; TDFA, thulium-doped fiber amplifier; SMF, single mode fiber; VOA, variable optical attenuator; OSA, optical spectrum analyzer. (b) The optical spectrum and (c) the autocorrelation trace of the compressed pump soliton at the input of the microtaper.

Fig. 3 (a) shows the experimental setup of the wavelength converter. Mode locked seed pulses are launched from a fiber laser (AdValue AP-ML) at a repetition rate of $30 \mathrm{MHz}$, duration of $900 \mathrm{fs}$, central wavelength of $1.938 \mu \mathrm{m}$ and average power of $7 \mathrm{~mW}$. The pulses are amplified and compressed in a pulse formatting stage consisting of a thulium-doped fiber amplifier followed by a $1.8 \mathrm{~m}$ long SMF-28. In the amplifier, the average power is increased to $40.5 \mathrm{~mW}$. The pulses emerge slightly chirped due to anomalous dispersion of the thulium-doped silica fiber. The pulses then propagate in the SMF-28 fiber and experiences compression due to 
higher-order soliton compression dynamics [31]. The pump pulses are spectrally and temporally characterized right after the pulse formatting stage using an OSA and autocorrelator. Fig. 3 (b) and (c) show the optical spectrum of the pump pulse and the corresponding autocorrelation trace. The two main lobes in the pump spectrum occurs due to the self-phase modulation in the SMF-28 fiber. The center part of the autocorrelation trace fits perfectly with a sech ${ }^{2}$ shape with a pulse width of $215 \mathrm{fs}$. In a more practical assembly, both the mode locked fiber laser and the pulse formatting stage could be replaced with a pulsed laser of similar output pulse characteristics.

After characterization, pump pulses from the formatting stage are delivered to a variable optical attenuator to control the pulse energy delivered to the microtaper. The frequency shifted output of the microwire is monitored using an OSA. It is worth mentioning that the whole system is entirely fiberized without using any free space optics. Furthermore, the low number of components adds to the simplicity and compactness of the setup. (a) Experiment

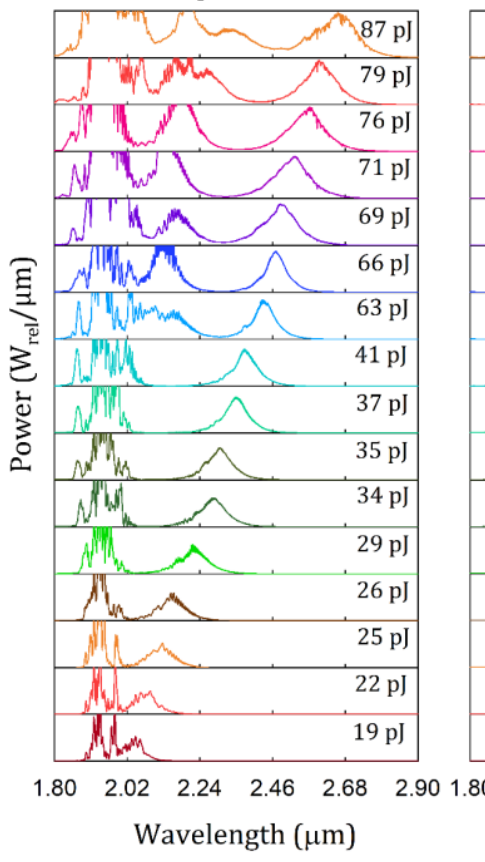

(b) Simulation

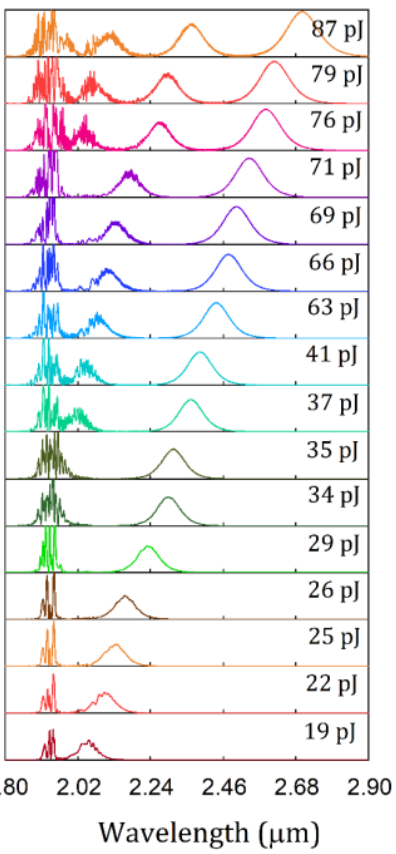

Fig. 4. (a) Measured output and (b) simulated spectra of the SSFS with increasing input pump pulse energy. The input pump energy is indicated for each spectrum.

Fig. 4 (a) shows experimental output spectra as a function of input pump energy, considering the total insertion loss of the taper. The calculated soliton number increases from 2.91 to 6.13 as the pump energy increases from $19 \mathrm{pJ}$ to $87 \mathrm{pJ}$. High-order solitons undergo temporal compression and break into multiple fundamental solitons due to higher order dispersion and Raman scattering. Raman scattering also leads to SSFS of the resulting solitons. Fig. 4 (a) shows that with the increase of pump energy, a soliton is clearly identifiable and shifts towards long wavelengths, reaching a maximum central wavelength of $2.667 \mu \mathrm{m}$ for a pump energy of $87 \mathrm{pJ}$ and average pump power of $2.6 \mathrm{~mW}$. This input energy can be further decreased by reducing the insertion loss of the SMF-taper-ChG fiber assembly by angle cleaving and applying anti-reflection coating at the fiber interfaces. With further increase in the pump energy, the main soliton shift slows down while the major fraction of the pump energy is transferred to secondary solitons and residual background.

To understand the combined effects of soliton compression, fission and SSFS, numerical simulations are performed by solving the nonlinear Schrödinger equation (NLSE) using split-step Fourier method, combined with adaptive step size [32], considering eight order of dispersion, Raman effect and self-steepening. In the simulation, polarized light and single mode propagation is considered. The wavelength dependence of waveguide nonlinearity is also included in the simulation. In the simulation, the 30 ps temporal window is discretized into $2^{12}$ samples for an input sech pulse of $215 \mathrm{fs}$ duration. The fractional contribution and the Raman response function are included with, $f_{r}=0.1, \tau_{1}=$ $15.2 \mathrm{fs}$ and $\tau_{2}=230.5 \mathrm{fs}$ [33]. In fig. 4 (b), it is seen that the center wavelength of the simulated Raman shifted pulse agrees well with the experiment.
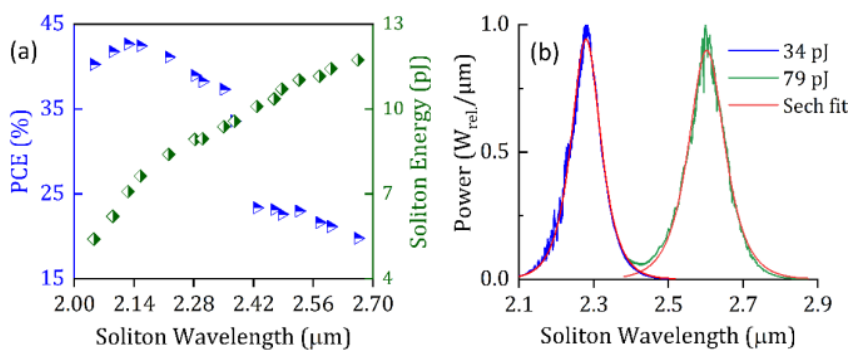

Fig. 5. (a) PCE and soliton energy as a function of soliton wavelength. (b) The spectrum of the fundamental soliton fitted with the spectrum of an ideal $\mathrm{sech}^{2}$ shaped pulse, at two different pump energies.

Fig. 5 (a) shows the PCE and energy of the Raman soliton as a function of center wavelength. PCE is defined as the ratio of the selfshifted soliton power to the total output power [17]. A maximum and minimum CE of $43 \%$ and $20 \%$ are obtained for a center wavelength of $2.127 \mu \mathrm{m}$ and $2.667 \mu \mathrm{m}$, respectively. The high PCE is ensured by the combination of the microwire specification and excitation conditions chosen to enhance Raman soliton formation, without the generation of broad supercontinuum. The initial increase in the CE with wavelength could be due to the incomplete shifting of the Raman soliton [16]. Any further increase in pump power leads to reduction in PCE, related to the generation of secondary solitons. A maximum pulse energy of $11.7 \mathrm{pJ}$ is obtained for the soliton centered at a wavelength of $2.667 \mu \mathrm{m}$.

The spectrum of the fundamental soliton fits well with a sech 2 spectrum (fig. 5 (b)), which is suggestive of transform limited pulses. For pulses centered at $2.280 \mu \mathrm{m}$ and $2.600 \mu \mathrm{m}$, the pulse durations are inferred to $62 \mathrm{fs}$ and $69 \mathrm{fs}$, respectively. This results in peak powers of $125 \mathrm{~W}$ and $144 \mathrm{~W}$, respectively.

The stability of the system is also evaluated by measuring the power and the center wavelength fluctuation. Fig. 6 shows the measured parameters of the soliton centered at $2.543 \mu \mathrm{m}$ for duration of an hour at an acquisition rate of $33 \mathrm{mHz}$. The calculated coefficient of variation (standard deviation/mean) of the power and the center wavelength are $2.8 \%$ and $0.57 \%$ respectively. Such numbers indicate a reasonable stability of the system. A stability analysis of the pump laser shows that it remains stable within a variation of $0.02 \%$ in power, and $0.03 \%$ in central wavelength. Simulations suggest that such fluctuations have a negligible impact 
on the resulting Raman soliton. Since the pump laser seems stable, then we assume that fluctuations observed of the Raman soliton may occur from gradual drift of polarization with time. The Raman soliton stability could be improved by replacing the silica fiber in the system by polarization maintaining fiber.
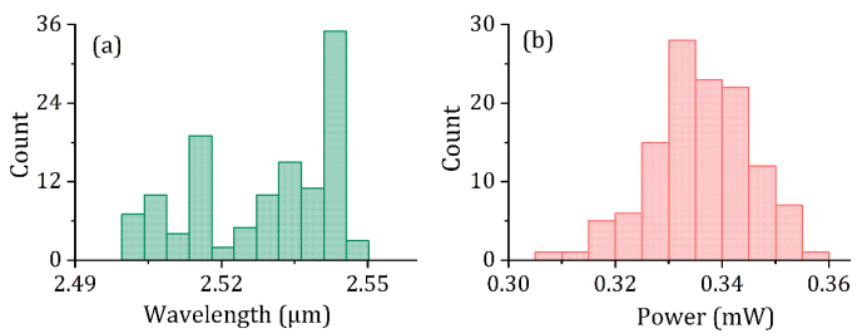

Fig. 6. The stability measurement of the (a) center wavelength and (b) power of the fundamental soliton.

Despite the generated SSFS up to a wavelength of $2.667 \mu \mathrm{m}$, we believe it is possible to further improve the current performance of this system in terms of CE and shifted wavelength that are currently limited by the generation of multiple solitons. It is well know that efficiency of SSFS can be improved by reducing the soliton number, thereby generating as few solitons as possible [34]. A pump source generating shorter pulses will reduce the soliton number as well increase the maximum frequency shift of the fundamental soliton since, $\Omega(z) \propto 1 / T_{0}^{4}$, where $\mathrm{T}_{0}$, represents the pump pulse duration [21]. Fig. 7 shows the simulation results for a pump duration of $90 \mathrm{fs}$ and peak power $540 \mathrm{~W}$. This results in $\mathrm{N}=3.64$ and yield a wavelength shift of the fundamental soliton up to $3.4 \mu \mathrm{m}$ for a propagation length of $8 \mathrm{~cm}$.

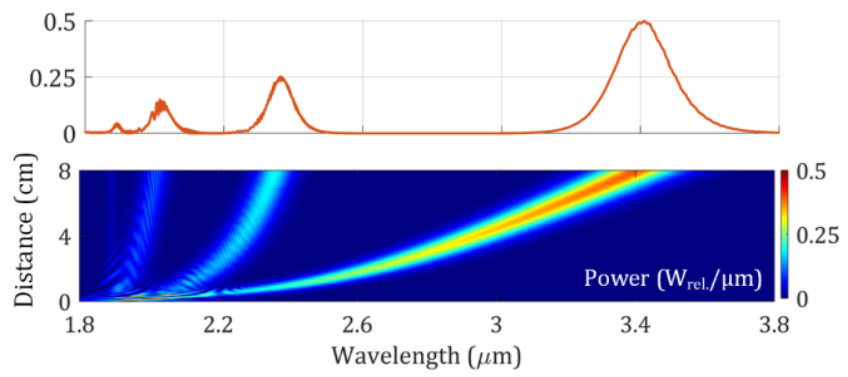

Fig. 7. Simulated spectral evolution of the pulse propagating in a $8 \mathrm{~cm}$ long ChG microwire.

In conclusion, we have demonstrated a wavelength converter tunable within the wavelength range of 2.047-2.667 $\mu \mathrm{m}$ in ChG microwire. The obtained PCE of $43 \%$ is the highest number ever reported on ChG glass. Applications of this compact MIR source include spectroscopic analysis and pollution monitoring.

Funding. Natural Sciences and Engineering Research Council of Canada.

\section{References}

1. A. Godard, Comptes Rendus Physique 8, 1100 (2007).

2. Y. Huang, J. Jivraj, J. Zhou, J. Ramjist, R. Wong, X. Gu, and V. X. D. Yang, Opt. Exp. 24, 16686 (2016).
3. D. M Bubb, J. S. Horwitz, R. A. McGill, D. B. Chrisey, M. R. Papantonakis, R. F. Haglund, Jr,and B. Toftmann, Appl. Phys. Lett. 79, 2847(2001).

4. P. Agostini and L. F DiMauro, Rep. Prog. Phys. 67, 813 (2004).

5. T. P. J. Blaikie, J. Couper, G. Hancock, P. L. Hurst, R. Peverall, G. Richmond, G. A. D. Ritchie, D. Taylor, and K. Valentine, Anal. Chem. 88, 11016 (2016).

6. L. Barreira, Y. Xue, G. Duporté, J. Parshintsev, K. Hartonen, M. Jussila, M. Kulmala, and M.-L. Riekkola, Atmos. Meas. Tech., 9, 3661 (2016).

7. P. M. Santos, E. R. Pereira-Filho, and L. E. Rodriguez-Saona J. Agric. Food Chem. 61, 1205 (2013).

8. P. Werle, F. Slemr, K. Maurer, R. Kormann, R. Mücke, and B. Jänker, Opt. Laser. Eng. 37, 101 (2002).

9. D. D. Hudson, S. Antipov, L. Li, I. Alamgir, T. Hu, M. El Amraoui, Y. Messaddeq, M. Rochette, S. D. Jackson, and A. Fuerbach, Optica 4, 1163 (2017).

10. C. R. Petersen, U. Møller, I. Kubat, B. Zhou, S. Dupont, J. Ramsay, T. Benson, S. Sujecki, N. Abdel-Moneim, Z. Tang, D. Furniss, A. Seddon and O. Bang. Nat. Photon 8, 830 (2014).

11. S. Venck, F. St-Hilaire, L. Brilland, A. N. Ghosh, R. Chahal, C. Caillaud, M. Meneghetti, J. Troles, F. Joulain, S. Cozic, S. Poulain, G. Huss, M. Rochette, J. M. Dudley, T. Sylvestre, Laser \& Photonics Reviews 14, 2000011 (2020)

12. I. Alamgir, F. St-Hilaire, and M. Rochette, Opt. Lett. 45, 857 (2020).

13. I. Alamgir, M. Rezaei, and M. Rochette, in OSA Advanced Photon, Congress (AP) 2020, paper NpM3E.4.

14. L. Li, N. Abdukerim, and M. Rochette Opt. Lett. 42, 639 (2017)

15. R. Kormokar, Md H. M. Shamim, and M. Rochette, J. Opt. Soc. Am. B 38, 466 (2021).

16. J. Wang, S. Lin, X. Liang, M. Wang, P. Yan, G. Hu, T. Albrow-Owen, S. Ruan, Z. Sun, and T. Hasan, Opt. Lett. 42, 3518 (2017).

17. J. Luo, B. Sun, J. Ji, E. L. Tan, Y. Zhang, and X. Yu, Opt. Lett. 42, 1568 (2017).

18. M. H. M. Shamim, I. Alamgir, and M. Rochette, in Conference on Lasers and Electro-Optics (2021), paper STh5A.2.

19. Y. Tang, L. G. Wright, K. Charan, T. Wang, C. Xu, and F. W. Wise, Optica 3, 948 (2016).

20. S. Duval, J.-C. Gauthier, L.-R. Robichaud, P. Paradis, M. Olivier, V. Fortin, M. Bernier, M. Piché, and R. Vallée, Opt. Lett. 41, 5294 (2016).

21. G. P. Agrawal, Nonlinear Fiber Optics, 5th ed. (Academic, 2013).

22. M. Yu. Koptev, E. A. Anashkina, A. V. Andrianov, V. V. Dorofeev, A. F. Kosolapov, S. V. Muravyev, and A. V Kim, Opt. Lett. 40, 4094 (2015).

23. R. E. Slusher, G. Lenz, J. Hodelin, J. Sanghera, L. B. Shaw, and I. D. Aggarwal, J. Opt. Soc. Am. B 21, 1146 (2004).

24. J. S. Sanghera, I. D. Aggarwal, L. B. Shaw, C. M. Florea, P. Pureza, V. Q. Nguyen, and F. Kung, Int. J. Appl. Glass Sci. 1, 296 (2010).

25. T. Cheng, Y. Kanou, K. Asano, D. Deng, M. Liao, M. Matsumoto, T. Misumi, T. Suzuki, and Y. Ohishi, Appl. Phys. Lett. 104, 121911 (2014).

26. E. C. Mägi, L. B. Fu, H. C. Nguyen, M. R. E. Lamont, D. I. Yeom, and B. J. Eggleton, Opt. Express 15, 10324 (2007).

27. L. Li, N. Abdukerim and M. Rochette, Optics Express 24(17), 18931 (2016).

28. A. Al-Kadry and M. Rochette, J. Lightwave Technol. 31, 1462 (2013).

29. A. Al-kadry and M. Rochette, J. Opt. Soc. Am. B 29, 1347 (2012).

30. I. Alamgir, N. Abdukerim, and M. Rochette, Opt. Lett. 44, 4467 (2019).

31. L. F. Mollenauer, R. H. Stolen, J. P. Gordon, and W. J. Tomlinson, " Opt. Lett. 8, 289-291 (1983).

32. O. V. Sinkin, R. Holzlohner, J. Zweck and C. R. Menyuk, J. of Lightwave Tech., 21, 61 (2003).

33. C. Xiong, E. Magi, F. Luan, A. Tuniz, S. Dekker, J. S. Sanghera, L. B. Shaw, I. D. Aggarwal, and B. J. Eggleton, " Appl. Opt. 48, 5467 (2009)

34. S. A. Dekker, A. C. Judge, R. Pant, I. Gris-Sánchez, J. C. Knight, C. M. de Sterke, and B.J. Eggleton, Opt. Express 19, 17766-17773 (2011). 